\title{
Linear time-varying Luenberger observer applied to diabetes
}

\author{
ONOFRE OROZCO-LÓPEZ , CARLOS E. CASTAÑEDA , AGUSTÍN RODRÍGUEZ-HERRERO , \\ GEMA GARCÍA-SÁEZ AND ELENA HERNANDO
}

\begin{abstract}
We present a linear time-varying Luenberger observer (LTVLO) using compartmental models to estimate the unmeasurable states in patients with type 1 diabetes. The LTVLO proposed is based on the linearization in an operation point of the virtual patient (VP), where a linear time-varying system is obtained. LTVLO gains are obtained by selection of the asymptotic eigenvalues where the observability matrix is assured. The estimation of the unmeasurable variables is done using Ackermann's methodology. The Lyapunov approach is used to prove the stability of the time-varying proposal. In order to evaluate the proposed methodology, we designed three experiments: A) VP obtained with Bergman's minimal model, B) VP obtained with Hovorka's model, and C) real patient data set. For both experiments A) and B), it is applied a meal plan to the VP, where the dynamic response of each state model is compared to the response of each variable of the time-varying observer. Once the observer is obtained in experiment $\mathrm{B}$ ), the proposal is applied to experiment $C$ ) with data extracted from real patients and the unmeasurable state space variables are obtained with the LTVLO. LTVLO methodology has the feature of being updated each time instant to estimate the states under a known structure. The results are obtained using simulation with $M a t l a b^{T M}$ and Simulink $^{T M}$. The LTVLO estimates the unmeasurable states from in silico patients with high accuracy by means of the update of Luenberger gains at each iteration. The accuracy of the estimated state space variables is validated through the fit parameter.
\end{abstract}

INDEX TERMS Artificial pancreas, Luenberger observer, type 1 diabetes, Glucose

\section{INTRODUCTION}

Diabetes is a chronic disease characterized by high levels of blood glucose (hyperglycemia). Type 1 diabetes mellitus (T1DM) occurs when the pancreas does not produce insulin, which is the key hormone of carbohydrate metabolism. $\beta$ cells response in a healthy person is characterized by reducing or increasing insulin secretion to match low or high blood glucose concentrations [1]. A hyperglycemic condition leads to serious damage on many of the body's systems in a medium-term, especially in nerves and blood vessels, developing retinopathy and nephropathy among others. Since 1920 insulin therapy has evolved bringing the ability to mimic the average physiological profile of insulin secretion with the aim of trying to regulate glucose levels [2]. T1DM is controlled by therapies based on the administration of exogenous insulin following multiple ( 3 - 5 per day) insulin injections (MDI) [3]. Nowadays, the use of insulin pumps makes possible the CSII therapy (Continuous Subcutaneous Insulin Infusion). CSII makes the treatment less invasive, reducing pain associated with MDI and can improve patient lifestyle [4]. This CSII therapy uses Lispro insulin (fast acting insulin).

Both therapies, MDI and CSII, need be complemented with 4-7 daily fingerstick to measure the glucose. The patient uses the measurements and his metabolic state to decide the insulin dose. This dose will keep patient in a safe metabolic state until the next decision. A painful consequence derived by MDI or CSII is when exogenous insulin is administered in excess, hypoglycaemia events appear, which is very dangerous to human health in short-term. Patients have to decide the insulin bolus to be administered before each carbohydrate (CHO) intake. Fitting this prandial bolus to the meal is a 
complex task, mainly due to the variability in insulin effects and the presence of CHOs with different absorption rates. People with T1DM might try to reduce the insulin doses to avoid hypoglycaemia, but increasing the hyperglycaemia risk.

The development of an artificial pancreas (AP) is a field of intensive research to optimize CSII therapy, to reduce the associated risks and to avoid the patient dependence of his illness, with the continuous take of decisions about his illness. An AP integrates three components: an insulin pump, a subcutaneous continuous glucose monitoring (CGM) system and a control algorithm connected by wireless communication [5]. The current challenges of the scientific community working on building the AP are: develop more reliable CGM sensors, create faster-acting insulins, administer the glucagon hormone to recover from hypoglycemia and design control algorithms. Control algorithms should be capable of dealing with physiological interpatient and intrapatient variability and working against delays in subcutaneous glucose measurement and insulin action [6]. In [7] Trevitt et al. defined three generations of devices. The first generation is not fully automated and it involves three concepts: 1) very-lowglucose insulin off pump, pump shuts off when user not responding to low-glucose alarm; 2) hypoglycaemia minimizer, predictive hypoglycaemia causes alarms and pump suspension; and 3) hypoglycaemia and hyperglycaemia minimizer, it is the previous system with a new feature to allow insulin dose when the glucose is above a threshold. The second generation includes closed-loop control and it involves two concepts: 1) automated basal and hybrid closed loop, the system is notified with meal information; and 2) fully automated closed-loop, the system does not use intake information. The third generation involves fully automated multi hormone closed-loop.

Mathematical modelling is a powerful tool that allows describing the behaviour of a dynamic system. In T1DM mathematical models emerge as an option to reduce the test time, allowing simulations that could be dangerous to health and to obtain inner variables. To T1DM there exist compartmental models based in Ordinary Differential Equations (ODEs) [8]-[11] and for Type 2 Diabetes mellitus has been proposed a model based in delay-Discrete Differential Equations (DDEs) [12].

In T1DM, it is possible to measure the glucose concentration and to know the insulin doses, the inner variables are not available for measurement, (i.e. the insulinemia). Different disturbances are taken into account by mathematical models for T1DM; some disturbances are: endogenous glucose production in the liver, renal excretion, insulin-independent glucose utilization (e.g. central nervous system) and ingested CHOs. Another disturbance is physical activity practising but it is still not considered on the mathematical models; other disturbances such as stress situations are not modelled as parametric variation [13].

On the other hand, it is known that in the design of some control algorithms, it is necessary the knowledge of the T1DM patient variables, which means that all state space variables are measurable; but in reality this is not possible. Independently of the model chosen for the control algorithm design, it is only possible to measure the subcutaneous glucose concentration considered as the output variable and to know the model input, that is, the insulin dose amount (basal and prandial). One alternative to approach this problem is the use of linear and nonlinear observers. Such is the case of the Luenberger observer applied to linear and non-linear systems [14]-[19]. Other observers use sliding modes [20]-[24]. Such as the ones applied to artificial pancreas [12], [25]-[28], among others. The strategies that use these observers result in obtaining constant gains, which in a general way constitutes a good alternative in order to estimate the unmeasurable variables.

In this paper, we propose to extend the theory of Luenberger observers for time-invariant systems, to a time-varying Luenberger observer. These time-varying systems result from the linearization of two T1DM nonlinear models. The aim of extending the theory of linear time-invariant systems to timevariant systems is to make the observer able to increase the precision in the estimation of unmeasurable variables of nonlinear systems. This type of linear time-varying Luenberger observer should be capable of estimating the unmeasurable variables with a structure of known models. The observer should be robust to deal with parametric variability in T1DM patients that will change within the day and also from day to day, due to lifestyle changes and metabolic performance [13]. The main disturbance to take into account is a meal intake, composed mainly of CHOs, which is usually used to stimulate the dynamic system of a T1DM patient. We design a Luenberger linear time-varying observer applied to the field of AP using Bergman's [8] and Hovorka's [10] compartmental models. The computation of the time-varying Luenberger gains is obtained by the correct selection of the asymptotic eigenvalues to assure convergence of the unmeasurable variables and Lyapunov stability proof is done. Different experiments are used to evaluate the methodology with VP and data extracted from insulin and glucose variables obtained from real patients. In order to validate the methodology, statistical analysis is done.

This paper is organized as follows: in section II the main theoretical information regarding the T1DM models as well as the state space observers are explained; in section III are explored the proposed methodologies for the time-varying formulation and the design of the experiments is described; in section IV the results of the present work are shown; some discussion is presented in section V; and finally, conclusion is presented in section VI.

\section{STATE OF ART}

T1DM patients can be described physiologically through mathematical models, whose input and output are the insulin infusion and the glucose concentration, respectively. In this work, a time-variant state space observer is designed for two mathematical models commonly used to describe T1DM 
patients.

\section{A. BERGMAN'S MODEL}

Bergman's minimal model contains three state space variables that describe the glucose-insulin regulatory system. With the purpose of facilitating the mathematical comprehension on later sections, in this work Bergman's model is taken from [8] and is arranged in state space form as follows:

$$
\begin{aligned}
& \dot{x}_{1}=-p_{1}\left[x_{1}-G_{b}\right]-x_{2} x_{1}+d \\
& \dot{x}_{2}=-p_{2} x_{2}+p_{3}\left[x_{3}-I_{b}\right] \\
& \dot{x}_{3}=-\eta\left[x_{3}-I_{b}\right]+\gamma\left[x_{1}-h\right]^{+} t+u_{i}
\end{aligned}
$$

where $x_{1}$ renames $G$ (blood glucose concentration) $(\mathrm{mg} / \mathrm{dl})$, $x_{2}$ corresponds to $X$ (insulin effect on glucose concentration reduction) $(1 / \mathrm{min})$, and $x_{3}$ replaces $I$ (insulin concentration in plasma) $(\mu U / m l)$. It is important to remark that for a T1DM patient, the rate of pancreatic $\beta$-cells release insulin is null $(\gamma=0)$; while in a healthy person does not exist exogenous insulin $\left(u_{i}=0\right)$.

The Table 1 describes: 1$)$ the parameters and values for three T1DM patients, 2) the mean of these three patients defined as VP\#0, and 3)the parameters and values of a virtual healthy person (VHP). The parameter settings were obtained from [29] to simulate in silico patients with Bergman's model (1).

For all patients, $G_{b}=70 \mathrm{mg} / \mathrm{dl}$ and $I_{b}=7 \mathrm{mU} / \mathrm{dl}$ are the basal glucose and basal insulin respectively.

\section{1) Generating VP with Bergman minimal model}

In [30] Berger and Rodbar modelled the course of insulin absorption which is basically responsible for the characteristic form of plasma profiles for different types of insulin and how its kinetic evolves. A mathematical representation of the profile that appears in blood after insulin is subcutaneously administered is presented as follows:

$$
u_{i}=u \frac{s \tau^{s} T_{50}^{s}}{\tau\left(T_{50}^{s}+\tau^{s}\right)^{2}},
$$

where $u$ is the insulin dose that has been injected as a bolus $(U I)$, which is represented as the amplitude of Dirac-delta; $T_{50}$ is time interval to permit $50 \%$ of the injected dose to be absorbed; $s$ is the parameter itself absorption type of insulin used, and $\tau$ is the time after injection.

In [31] Lehmann and Deutsch modelled CHOs absorption via intestine as $d$, which is considered the main disturbance of the model, using the following function:

$$
d=D_{G} \frac{r_{1} r_{2}}{r_{2}-r_{1}}\left(e^{-r_{1} t}-e^{-r_{2} t}\right),
$$

where $D_{G}$ is the amount of ingested carbohydrates ( $g$ of CHOs $), r_{1}$ and $r_{2}(1 / \mathrm{min})$ are specific parameters for the type of CHOs selected to represent a slow rate of assimilation.

The equations (2)-(3) are absorption functions added to Bergman's model, which shape the absorption of insulin administered as a bolus and CHOs ingested amount (both depicted as delta signals in simulation), respectively to in silico patients. The absorption functions to generate a VP with Bergman's model have the next set of parameters in equations (2)-(3): $s=1.7 ; a=0.02$ (unitless) and $b=1$ (unitless) are natural constants of the Lispro insulin; $r_{1}=0.0170(1 / \mathrm{min})$ and $r_{2}=0.173(1 / \mathrm{min})$ are parameters of $\mathrm{CHO}$ absorption [32].

\section{B. HOVORKA'S MODEL}

The model was built based on experimental and modeling work, which employed glucose tracers to determine structure and parameter values of glucose kinetics [33]. Hovorka's model described in [10] and [34] consists of a glucose subsystem (glucose absorption, distribution and disposal), an insulin subsystem (insulin absorption, distribution and disposal), and an insulin action subsystem (insulin action on glucose transport, disposal and endogenous production). With the aim of using a common nomenclature, Hovorka's model is rewritten in terms of $x_{i}$ as follows:

$$
\begin{aligned}
& \dot{x}_{1}=k_{a}\left[G(t)-x_{1}\right] \\
& \dot{x}_{2}=-\left[\frac{f_{01}^{\circ}}{V_{G} G(t)}+x_{4}\right] x_{2}+k_{12} x_{3}-E G P_{0} x_{6}+d \\
& \dot{x}_{3}=x_{4} x_{2}-\left[k_{12}+x_{5}\right] x_{3} \\
& \dot{x}_{4}=-k_{a 1} x_{4}+k_{b 1} x_{7} \\
& \dot{x}_{5}=-k_{a 2} x_{5}+k_{b 2} x_{7} \\
& \dot{x}_{6}=-k_{a 3} x_{6}+k_{b 3} x_{7} \\
& \dot{x}_{7}=\frac{x_{8}}{V_{I} t_{\max , I}}-k_{e} x_{7} \\
& \dot{x}_{8}=\frac{x_{9}}{t_{\max , I}}-\frac{x_{8}}{t_{\max , I}} \\
& \dot{x}_{9}=u-\frac{x_{9}}{t_{\max , I}}
\end{aligned}
$$

where $x_{1}$ represents $C$ and is glucose concentration $(\mathrm{mmol} / \mathrm{l})$ in the subcutaneous tissue. This submodel of the interstitial glucose kinetics was added from [34]. $x_{2}$ replaces $Q_{1}$ and represents the mass of glucose in the accessible compartment $(\mathrm{mmol}) ; x_{3}$ rewrites $Q_{2}$ that is the non-accessible glucose mass compartment $(\mathrm{mmol}) ; x_{4}$ corresponds to $X_{1}$ and is the remote effect of insulin on glucose distribution/transport $(1 / \mathrm{min}) ; x_{5}$ represents $X_{2}$ and is the remote effect of insulin on glucose disposal $(1 / \mathrm{min}) ; x_{6}$ renames $X_{3}$ that is the remote effect of insulin on endogenous glucose production $(1 / \mathrm{min}) ; I$ is changed by $x_{7}$ and is the plasma insulin concentration $(m U / d l) ; x_{8}$ and $x_{9}$ are interchanged with $S_{2}$ and $S_{1}$, respectively, and are two-compartments chain representing absorption of subcutaneously administered short-acting insulin $(m U) ; G(t)=x_{2} / V_{G}$ is the glucose concentration in the plasma $(\mathrm{mmol} / \mathrm{l}) ; u$ is the insulin dose $(m U)$. The inner and outer disturbances are expressed as:

$$
d=U_{G}(t)+E G P_{0}-f_{R}-f_{01}^{c},
$$

where $E G P_{0}$ represents the endogenous glucose production extrapolated to a 0 concentration $(\mathrm{mmol} / \mathrm{min})$, and $f_{R}$ is the renal glucose clearance $(\mathrm{mmol} / \mathrm{min})$ above the glucose threshold of $9 \mathrm{mmol} / \mathrm{l}$ represented as:

$f_{R}=\left\{\begin{array}{ccc}0.003(G(t)-9) V_{G} & \text { if } & G(t) \geq 9 \mathrm{mmol} / l \\ 0 & \text { otherwise }\end{array}\right.$ 


\begin{tabular}{|c|c|c|c|c|c|c|}
\hline \multirow{2}{*}{$\begin{array}{l}\text { Symbol } \\
\text { / Units }\end{array}$} & \multirow[t]{2}{*}{ Description } & \multicolumn{5}{|c|}{$\begin{array}{l}\text { Value per patient } \\
\end{array}$} \\
\hline & & VHP & VP\#0* & VP\#1 & VP\#2 & VP\#3 \\
\hline $\begin{array}{c}p_{1} \\
(1 / \min )\end{array}$ & $\begin{array}{l}\text { Insulin independent } \\
\text { rate constant }\end{array}$ & 0.0317 & 0 & 0 & 0 & 0 \\
\hline $\begin{array}{c}p_{2} \\
(1 / \min )\end{array}$ & $\begin{array}{l}\text { Rate for decrease } \\
\text { in tissue glucose } \\
\text { uptake ability }\end{array}$ & 0.0123 & 0.0112 & 0.0123 & 0.0072 & 0.0142 \\
\hline $\begin{array}{c}p_{3} \\
{\left[\frac{(\mu U / m l)}{m i n^{2}}\right]}\end{array}$ & $\begin{array}{l}\text { Insulin-dependent } \\
\text { increase }\end{array}$ & $8.2 \times 10^{-8}$ & $4.48 \times 10^{-\varepsilon}$ & $8.2 \times 10^{-8}$ & $3.6 \times 10^{-8}$ & $1.656 \times 10^{-6}$ \\
\hline$\left[\begin{array}{c}\gamma \\
\frac{(\mu U / m l)}{\min ^{2}(m g / d l)}\end{array}\right]$ & $\begin{array}{l}\text { Rate of the } \\
\text { pancreatic } \beta \text {-cells } \\
\text { release insulin }\end{array}$ & $6.5 \times 10^{-5}$ & 0 & 0 & 0 & 0 \\
\hline$\underset{(1 / \min )}{\eta}$ & $\begin{array}{l}\text { First order decay } \\
\text { rate for insulin } \\
\text { in blood }\end{array}$ & 0.2659 & 0.2646 & 0.2659 & 0.2465 & 0.2814 \\
\hline $\begin{array}{c}h \\
(m g / d l)\end{array}$ & $\begin{array}{l}\text { Glucose threshold } \\
\text { above } \beta \text {-cells } \\
\text { release insulin }\end{array}$ & 79.0353 & 0 & 0 & 0 & 0 \\
\hline
\end{tabular}

with $f_{01}^{c}(\mathrm{mmo} / \mathrm{min})$ as the total non-insulin-dependent glucose flux corrected by the ambient glucose concentration represented as:

$$
f_{01}^{c}=\left\{\begin{array}{ccc}
f_{01} & \text { if } & G(t) \geq 4.5 \mathrm{mmol} / \mathrm{l} \\
f_{01} \frac{G(t)}{4.5} & & \text { otherwise }
\end{array} .\right.
$$

The Glucose absorption is a fundamental process affecting postprandial glucose excursions. In Hovorka's model (4), the gut absorption rate $U_{G}(t)(\mathrm{mg} / \mathrm{min})$ is represented by:

$$
U_{G}(t)=\frac{D_{G} A_{G} \tau e^{\frac{-\tau}{t_{\max , G}}}}{t_{\max , G}},
$$

where $t_{\max , G}$ is the time-of-maximum of CHO absorption, $D_{G}$ is the amount ( $\mathrm{mg}$ ) of CHOs ingested, $A_{G}$ is carbohydrate bioavailability (unitless).

Table 2 includes the parametric values of six Hovorka patients (VP\#1 - VP\#6) and a VP\#0, whose parameters are computed as the mean and standard deviation of the other 6 patients [33].

The parameters plasma insulin elimination rate $k_{e}=$ $0.138(1 / \mathrm{min})$, CHOs bioavailability $A_{G}=0.8$ (unitless), time-of-maximum absorption of CHOs $t_{\max , G}=40(\mathrm{~min})$ and time-to-maximum absorption of subcutaneously injected short-acting insulin $t_{\max , I}=55(\mathrm{~min})$ are equal for the six patients and VP\#0.

\section{METHODOLOGY}

In this section, the proposed method is described in generic terms, where the time-varying observer design and its implementation are presented. Additionally, it is shown the Lyapunov approach in order to prove the stability of the timevarying proposal.

\section{A. LINEAR TIME-VARYING LUENBERGER OBSERVER DESIGN}

In order to introduce the proposed method, we consider a class of continuous-time nonlinear Single-Input-Single-
Output (SISO) disturbed system, which represents the structure of Bergman's and Hovorka's compartmental models, as follows:

$$
\begin{aligned}
& \dot{x}=f(x)+g u+d, \\
& y=h(x),
\end{aligned}
$$

where $x \in \Re^{n \times 1}$ is the state space vector, $u$ is the input signal; $y \in \Re^{1 \times n}$ represents the output of the system, with $n$ as the state dimension; $f(x)$ constitute smooth vector function; $g$ is the term that determines an affine control; $d$ represents the vector function of external and internal disturbance; $h(x)$ is a smooth function continuous and differentiable. For both afformentioned models (Bergman (1) and Hovorka (4)), $u$ is the insulin infusion dose; the output signal in Bergman's model (1) $y=x_{1}=G$ is the plasma glucose concentration and in Hovorka's model (4) $y=x_{1}=C$ is the interstitial glucose concentration measured with CGM sensor. Then, the system (9)-(10) is linearized using the Jacobian in the following form [35]:

$$
A=\left.\frac{\partial f(x)}{\partial x}\right|_{x=\rho}, b=\left.\frac{\partial g}{\partial u}\right|_{x=\rho}, c=\left.\frac{\partial h(x)}{\partial x}\right|_{x=\rho} .
$$

In order to avoid the loss of information by the linearization process, the operation point $\rho$ used to evaluate the resulting matrices from the Jacobian, is going to be updated with the new values of state variables at each time instant $(t)$, resulting in the following linear time-varying system:

$$
\begin{aligned}
& \dot{x}=A(t) x+b u+d, \\
& y=c x,
\end{aligned}
$$

where $A(t)$ is the time-varying state matrix containing the dynamic information of linearized state space variables; $b$ is the input vector, $u$ is the control signal; $c$ is the ouput vector. For this particular application $d$ is considered as disturbance due to real amount of CHOs intake.

The conventional Luenberger observer has been a popular approach to state estimation for linear dynamical systems. 
TABLE 2. Hovorka's model parameter values and description.

\begin{tabular}{|c|c|c|c|c|c|c|c|c|}
\hline \multirow{2}{*}{$\begin{array}{c}\text { Symbol } \\
\text { / units }\end{array}$} & \multirow[t]{2}{*}{ Description } & \multicolumn{7}{|c|}{ Value per patient } \\
\hline & & $\mathrm{VP \# 0^{* }}$ & VP\#1 & VP\#2 & VP\#3 & VP\#4 & VP\#5 & VP\#6 \\
\hline $\begin{array}{c}k_{12} \\
(1 / \min )\end{array}$ & Transfer rate & 0.066 & 0.0343 & 0.0871 & 0.0863 & 0.0968 & 0.0390 & 0.0458 \\
\hline $\begin{array}{c}k_{a 1} \\
(1 / \min )\end{array}$ & Deactivation rate & 0.006 & 0.0031 & 0.0157 & 0.0029 & 0.0088 & 0.0007 & 0.0017 \\
\hline $\begin{array}{c}k_{a 2} \\
(1 / \min )\end{array}$ & Deactivation rate & 0.06 & 0.0752 & 0.0231 & 0.0495 & 0.0302 & 0.1631 & 0.0689 \\
\hline $\begin{array}{c}k_{a 3} \\
(1 / \min )\end{array}$ & Deactivation rate & 0.03 & 0.0472 & 0.0143 & 0.0691 & 0.0118 & 0.0114 & 0.0285 \\
\hline $\begin{array}{c}V_{I} \\
(l / k g)\end{array}$ & $\begin{array}{l}\text { Insulin volume } \\
\text { distribution }\end{array}$ & 0.12 & 0.18 & 0.13 & 0.22 & 0.14 & 0.14 & 0.13 \\
\hline $\begin{array}{c}V_{G} \\
(l / k g)\end{array}$ & $\begin{array}{l}\text { Glucose volume } \\
\text { distribution }\end{array}$ & 0.16 & 0.18 & 0.13 & 0.22 & 0.14 & 0.14 & 0.13 \\
\hline $\begin{array}{c}k_{b 1} \\
(1 / \min )\end{array}$ & $\begin{array}{l}\text { Insulin elimination } \\
\text { rate }\end{array}$ & $\begin{array}{r}3.584 \\
* 10^{-9}\end{array}$ & $\begin{array}{r}2.076 \\
* 10^{-9}\end{array}$ & $\begin{array}{r}6.932 \\
* 10^{-9}\end{array}$ & $\begin{array}{l}2.9435 \\
* 10^{-9}\end{array}$ & $\begin{array}{r}9.471 \\
* 10^{-9}\end{array}$ & $\begin{array}{r}4.6457 \\
* 10^{-10}\end{array}$ & $\begin{array}{l}1.0823 \\
* 10^{-9}\end{array}$ \\
\hline $\begin{array}{c}k_{b 2} \\
(1 / \min )\end{array}$ & $\begin{array}{l}\text { Insulin elimination } \\
\text { rate }\end{array}$ & $\begin{aligned} & 5.74 \\
* & 10^{-9}\end{aligned}$ & $\begin{array}{r}1.541 \\
* 10^{-9}\end{array}$ & $\begin{array}{c}3.327 \\
* 10^{-9}\end{array}$ & $\begin{array}{l}1.2437 \\
* 10^{-8}\end{array}$ & $\begin{array}{l}1.7743 \\
* 10^{-9}\end{array}$ & $\begin{array}{l}2.2875 \\
* 10^{-8}\end{array}$ & $\begin{array}{l}5.0527 \\
* 10^{-9}\end{array}$ \\
\hline $\begin{array}{c}k_{b 3} \\
(1 / \min )\end{array}$ & $\begin{array}{l}\text { Insulin elimination } \\
\text { rate }\end{array}$ & $\begin{array}{c}1.82 \\
* 10^{-7}\end{array}$ & $\begin{array}{l}4.3112 \\
* 10^{-7}\end{array}$ & $\begin{array}{l}1.2797 \\
* 10^{-7}\end{array}$ & $\begin{array}{l}4.9925 \\
* 10^{-7}\end{array}$ & $\begin{array}{c}1.062 \\
* 10^{-7}\end{array}$ & $\begin{array}{l}1.0042 \\
* 10^{-7}\end{array}$ & $\begin{array}{r}7.695 \\
* 10^{-8}\end{array}$ \\
\hline${ }_{E G P_{0}}$ & EGP extrapolated & & & & & & & \\
\hline $\begin{array}{l}(\mathrm{mmol} / \mathrm{kg} \\
1 / \mathrm{min})\end{array}$ & $\begin{array}{l}\text { to } 0 \text { insulin } \\
\text { concentration }\end{array}$ & 0.0161 & 0.0148 & 0.0143 & 0.0156 & 0.0213 & 0.0200 & 0.0105 \\
\hline $\begin{array}{c}F_{01} \\
(\mathrm{mmol} / \mathrm{kg} \\
1 / \mathrm{min})\end{array}$ & $\begin{array}{c}\text { Glucose flux } \\
\text { non insulin } \\
\text { dependent }\end{array}$ & 0.0097 & 0.0121 & 0.0075 & 0.0103 & 0.0119 & 0.0071 & 0.0092 \\
\hline $\begin{array}{l}I C R \\
(U / g)\end{array}$ & $\begin{array}{l}\text { Insulin-CHOs } \\
\text { ratio }\end{array}$ & 0.1 & 0.13 & 0.1 & 0.12 & 0.1 & 0.07 & 0.12 \\
\hline $\begin{array}{c}B I R \\
(U / h r)\end{array}$ & $\begin{array}{l}\text { Basal insulin } \\
\text { ratio }\end{array}$ & 0.8 & 0.89 & 0.95 & 0.9 & 1.05 & 1.2 & 1.1 \\
\hline
\end{tabular}

In the well known linear Luenberger observer, the output of the system (subcutaneous glucose measurement) is compared with the estimated output by the observer (estimated glu$\operatorname{cose}$ ). With the estimated state vector $\hat{x}$, the output estimation error $(e=y-c \hat{x})$ is calculated. The estimation error passing through an $\mathrm{n} \times 1$ constant gain vector $l$ is used as correcting term, in order to estimate the state $x$ of system (12)-(13) [36]. If the gain $l$ is properly designed, the difference will drive the estimate state to the actual state. Such estimator is called an asymptotic estimator and is described by the following equation:

$$
\dot{\hat{x}}=A \hat{x}+b u+d_{1}+l(y-c \hat{x}),
$$

where $\hat{x}=\left[\begin{array}{llll}\hat{x}_{1} & \hat{x}_{2} & \cdots & \hat{x}_{n}\end{array}\right]^{\top}$ is the estimated state vector and $d_{1}$ is the meal absorption used in the observer. The idea of this observer is to compute the constant gain vector $l$ such that all eigenvalues of matrix $[A-l c]$ from equation (14), can be set arbitrarily in the left-half of the complex plane using Ackermann's methodology. This can be done if and only if the pair $(A, c)$ of system (12)-(13) is observable [37].

In the present paper, the linear Luenberger observer theory for time-invariant systems is extended for a linear timevarying Luenberger observer. These time-varying systems result from the linearization of two T1DM nonlinear models (Bergman and Hovorka) considered in this work.

In order to estimate the dynamic of nonlinear system (9)(10) and including the available information about external and internal disturbances $d$, we propose to extend the linear time-invariant theory from equation (14) designing a timevarying Luenberger observer for a system with the form (12)(13), as:

$$
\dot{\hat{x}}=A(t) \hat{x}+b u+d_{1}+l(t)(y-c \hat{x}),
$$

where $A(t)$ is the linear time-varying state matrix, $b$ is the input vector, $d_{1}$ are the general disturbances used in the observer, $l(t)$ is the Luenberger's time-varying gain vector, which includes the time-varying feature of the present proposal, and $(y-c \hat{x})$ is the estimation error computed as the difference between measurable output $y$ and estimated output $c \hat{x}$.

The procedure to obtain the time-varying formulation consists of two stages, which are explained in the flow diagram presented in Figure 1. In this diagram, the block enclosed by short dashed line corresponds to Stage I, where it is tested the observability condition; this stage is executed only one time at beginning of the process. The block enclosed by long dashed line corresponds to Stage II, where it is obtained the estimation of state space variables, this stage is executed as infinite loop. These stages are detailed as follows:

Stage I. It is proved the observability property of system (12) at time instant $t_{0}$ :

a) The system (9)-(10) is linearized around an operation point $\rho$, through Jacobian at time $t_{0}$. The operation point $\rho$ is selected when output $y=x_{1}=90 \mathrm{mg} / \mathrm{dl}$ and the other state variables values are extracted to evaluate the Jacobian (11). 


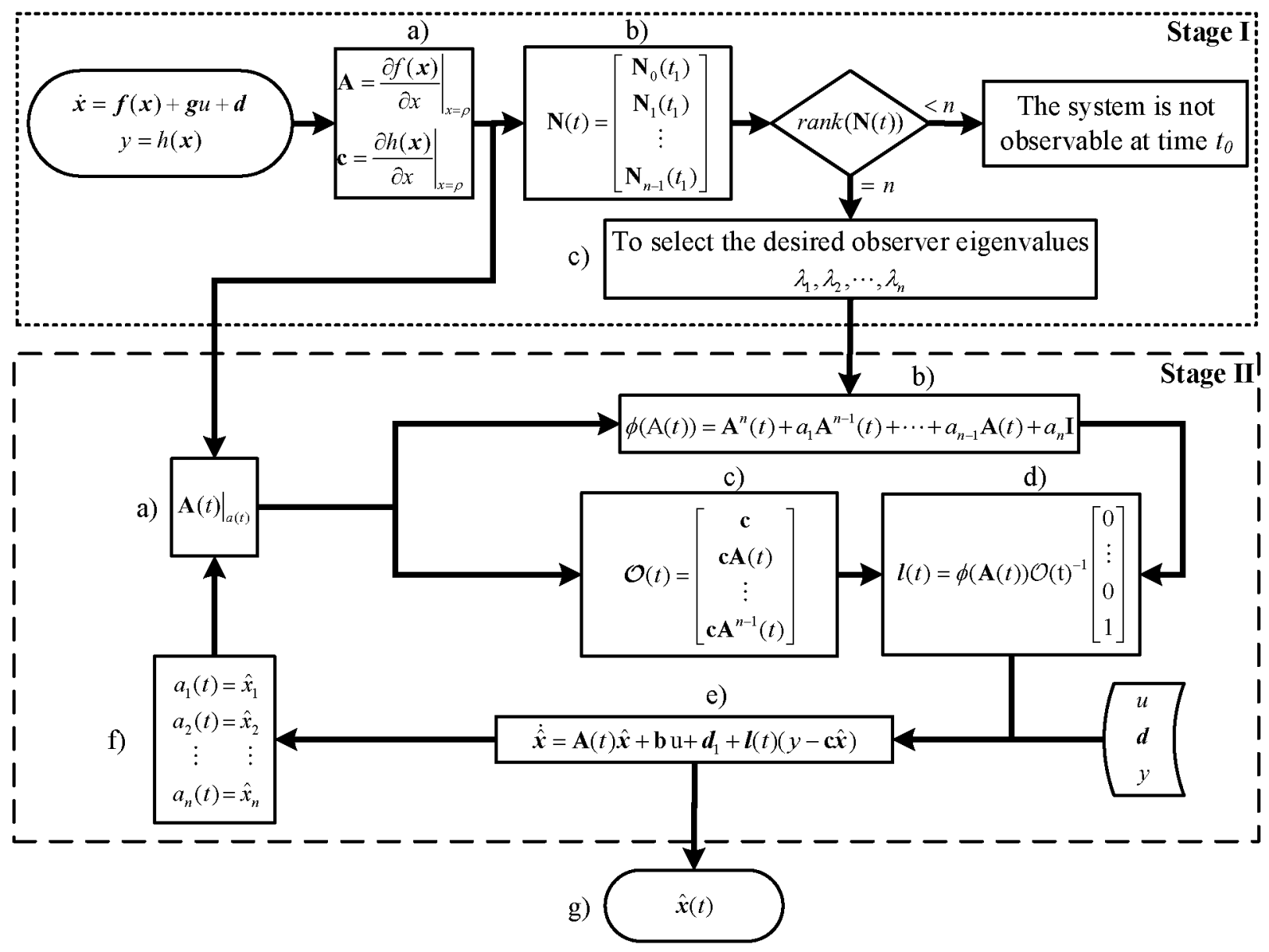

FIGURE 1. Luenberger time-varying state space estimator.

b) It is proved the observability condition in $t_{0}$ of system (12)-(13): the $\mathbf{n}$-dimensional pair $(A(t), c)$ is observable at $t_{0}$ if there exists a finite interval of time $t_{1}>t_{0}$ such that:

$$
\operatorname{rank}\left[\begin{array}{c}
\mathbf{N}_{0}\left(t_{1}\right) \\
\mathbf{N}_{1}\left(t_{1}\right) \\
\vdots \\
\mathbf{N}_{n-1}\left(t_{1}\right)
\end{array}\right]=n,
$$

where

$$
N_{q+1}(t)=N_{q}(t) \mathbf{A}(t)+\frac{d}{d t} N_{q}(t), q=0,1, \cdots, n-1,
$$

with

$$
\mathbf{N}_{0}(t)=c .
$$

Thus, if condition (16) holds, then the SISO system (12)-(13) is observable at time $t_{0}$. This means that for any unknown initial state $x_{0}$, there exists a finite $t_{1}>t_{0}$ such that it is possible to determine the initial state $x_{0}$ with the knowledge of the input $u$ (basal and prandial insulin) and the output $y$ (glucose). Otherwise system (12)-(13) is said to be unobservable at time $t_{0}$ [36].

c) The desired observer eigenvalues $\lambda_{1}, \lambda_{2}, \ldots, \lambda_{n}$ are selected to be located to the left of the eigenvalues of matrix $A$ at $t=0$, in the left-half of the complex plane. With this, the negative eigenvalues regulate the exponential rate of decay of estimation error and it is guaranteed the asymptotic convergence of the timevarying observer at time instant $t_{1}>t_{0}$. Notice that the desired eigenvalues can be set in the real axis or complex conjugate.

Stage II. To compute the time-varying Luenberger gains vector, in order to estimate the unmeasurable state space variables:

a) The matrix A obtained from process (11) becomes timevarying $\mathbf{A}(t)$ on Stage II, as it was described before; this is evaluating the matrix $\mathbf{A}$ at each time instant with the current values of $a(t)$. 
b) The desired characteristic polynomial is obtained through the desired observer eigenvalues as follows:

$$
\begin{aligned}
\phi(s) & =\left(s-\lambda_{1}\right)\left(s-\lambda_{2}\right) \cdots\left(s-\lambda_{n}\right) \\
& =s^{n}+\alpha_{1} s^{n-1}+\cdots+\alpha_{n-1} s+\alpha_{n},
\end{aligned}
$$

where $\lambda_{1}, \lambda_{2}, \ldots, \lambda_{n}$ represent the desired observer eigenvalues, and $\alpha_{1}, \alpha_{2}, \cdots, \alpha_{n}$ are used to compute $\phi(A(t))$ as follows:

$\phi(A(t))=A^{n}(t)+\alpha_{1} A^{n-1}(t)+\cdots+\alpha_{n-1} A(t)+\alpha_{n} I$,

where $I$ is the $n \times n$ identity matrix.

c) Once the observability condition is fulfilled at time instant $t>t_{0}$ with condition (16), it is necessary to compute the time-varying observability matrix at $t>t_{0}$. The compute of time-varying observability matrix is done at each time instant follows:

$$
\mathcal{O}(t)=\left[\begin{array}{c}
\mathrm{c} \\
\mathbf{c A}(t) \\
\vdots \\
\mathbf{c A}^{n-1}(t)
\end{array}\right] .
$$

For the particular cases treated in this work, it is assumed that observability matrix (21) is invertible at each time instant.

d) The required $l(t)$ Luenberger gains vector in (15) is determined by Ackermann's methodology, which is extended to time-varying case as:

$$
l(t)=\phi(A(t)) \mathcal{O}(t)^{-1}\left[\begin{array}{c}
0 \\
\vdots \\
0 \\
1
\end{array}\right] .
$$

e) The $l(t)$ Luenberger time-varying gains are used as correcting term in order to update the estimated state variables $\hat{x}$ at time instant $t$, where the estimation error $\left(y-\hat{x}_{1}\right)$ converges to 0 .

f) The estimated state space variables $\hat{x}$ are employed to update the time-varying terms $\left(a_{1}(t), a_{2}(t), \ldots, a_{n}(t)\right)$ in matrix $\mathbf{A}(t)$ on the next iteration.

g) Finally, the estimated state space variables are available.

\section{B. LINEAR TIME-VARYING LUENBERGER OBSERVER IMPLEMENTATION}

The observer goal is to estimate asymptotically the unmeasurable state space variables $\hat{x}$, through the knowledge of the output $y$ (subcutaneous glucose measurement) and the input $u$ (insulin). In this subsection, it is considered the observer implementation for Bergman (1) and Hovorka (4) models. This implementation is the procedure explained in Stages I and II presented in subsection III-A. For this, we define the estimation error as follows:

$$
e=x-\hat{x},
$$

and its dynamics is described as:

$$
\dot{e}=\dot{x}-\dot{\hat{x}} .
$$

Then, substituting equations (12) and (15) into (24), yields:

$$
\begin{aligned}
\dot{e} & =A(t) x+b u+d-A(t) \hat{x}-b u-d_{1}-l(t)(y-c(\hat{x})) \\
& =[A(t)-l(t) c](x-\hat{x})+\left(d-d_{1}\right) .
\end{aligned}
$$

Thus

$$
\dot{e}=[A(t)-l(t) c] e+\left(d-d_{1}\right) .
$$

Assumption 1: The linearized system obtained in (12)-(13) is observable at each time instant $t>t_{0}$.

Assumption 2: The time-varying observability matrix (21) at time instant $t>t_{0}$ is nonsingular.

The elements of matrix $A(t)$ that are time-varying correspond to the values, which are time-updated from estimated states in each instant $t>t_{0}$ by the asymptotic Luenberger observer.

Assumption 3: The matrices $A(t)$ and $\mathcal{O}(t)$ are Hurwitz at each simulation time instant. Then, the time-varying observability matrix (21) at time instant $t>t_{0}$ is nonsingular and it is possible to compute the timevarying $l(t)$ Luenberger gains, which are obtained in each instant $t>t_{0}$ using the Ackermann's methodology shown in (22).

Theorem 1: For system (12)-(13), the time-varying Luenberger observer (15) obtained from the Ackermann's methodology (22), ensures that the estimation error (23) is semi globally uniformly ultimately bounded (SGUUB).

The proof of Theorem 1 is presented in Appendix A.

The procedure of designing the LTVLO is going to be implemented below. For the case of nonlinear Bergman's model (1) with the form (9)-(10), it is applied the linearization procedure (11). In this procedure, the resulting matrix $A(t)$ and vector $c$ are:

$$
\mathbf{A}(t)=\left[\begin{array}{ccc}
\varrho_{1}(t) & \varrho_{2}(t) & 0 \\
0 & -p_{2} & p_{3} \\
0 & 0 & -\eta
\end{array}\right], c=\left[\begin{array}{lll}
1 & 0 & 0
\end{array}\right],
$$

where $\varrho_{1}(t)=-\hat{x}_{2}, \varrho_{2}(t)=-\hat{x}_{1}$ and the parameters are described in Table 1 . Note that the time-varying values $\varrho_{1}(t)$ and $\varrho_{2}(t)$ in (27) involve the estimated variables $\hat{x}_{1}$ and $\hat{x}_{2}$, which updates their values for the next iteration. These estimated variables give the feature of linear time-varying to the present proposal. As it was explained before, vector $c$ is time-invariant due to the output $y=x_{1}=G$ of system (1), which constitutes the plasma glucose concentration. Once the linearized procedure is done, it is proved the observability condition (16) at time instant $t_{0}$ of the pair $(A(t), c)(27)$ as:

$$
\operatorname{rank}\left[\begin{array}{ccc}
1 & \varrho_{2}(t) & \varrho_{2}^{2}(t)+p_{2} \varrho_{2}(t)+p_{3}\left[I_{b}-\varrho_{3}(t)\right] \\
0 & \varrho_{1}(t) & \varrho_{1}(t)\left[2 \varrho_{2}(t)-p_{1}-p_{2}\right]-p_{1} G_{b} \\
0 & 0 & -p_{3} \varrho_{1}(t)
\end{array}\right]^{\top}=3,
$$

where $\varrho_{3}(t)=\hat{x}_{3}$. The evaluation of (28) is only done in $t_{0}$ to prove the observability condition of the pair $(A(t), c)$. As 
the diagonal of matrix (28) depends on $\varrho_{1}(t)$ (say $\hat{x}_{1}$ ), and as $\hat{x}_{1} \neq 0$ due to system (1) the glucose can not be null, so the eigenvalues are chosen to avoid that this variable $\left(\hat{x}_{1}\right)$ does not cross by zero during the transitory period. As zero crosses by $\varrho_{1}(t)$ do not exist, the condition (16) in matrix (28) is achieved (rank of matrix $(28)=3$ ). Therefore, the linearized system with the form (12)-(13) obtained from system (1) with the pair $(A(t), c)$ (27) is observable at time $t_{0}$.

Next, the desired observer eigenvalues are adjusted to be located in the left-half of the complex plane. In a general way, it is recommendable that these eigenvalues are located to the left of the eigenvalues of matrix $A(t)$ at $t_{0}$. This results in a good practice in order to assure faster convergence of the observer and to consider the system response is dominated by the observer eigenvalues.

With the desired observer eigenvalues chosen as: $\lambda_{1}=$ $-2.659, \lambda_{2}=-0.126$ and $\lambda_{3}=-0.12$, it is obtained the characteristic polynomial of the form (19). The matrix $\phi(A(t))$ of the form (20) is computed each time instant, in such manner that the time-varying observability matrix (21) is:

$$
\mathcal{O}(t)=\left[\begin{array}{ccc}
1 & 0 & 0 \\
\varrho_{2}(t) & \varrho_{1}(t) & 0 \\
a_{2}^{2}(t) & \varrho_{1}(t)\left[\varrho_{2}(t)-p_{2}\right] & p_{3} \varrho_{1}(t)
\end{array}\right] .
$$

Then, it is computed the time-varying Luenberger gain vector $l(t)(22)$ as:

$$
l(t)=\left[\begin{array}{c}
2.905+\eta-p_{2}-\varrho_{1}(t) \\
-\frac{2.905 \eta-2.905 p_{2}-p_{2}\left(\eta-p_{2}\right)+\eta^{2}+0.6692}{\varrho_{2}(t)} \\
-\frac{(25 \eta+3)\left(200 \eta^{2}+557 \eta+67\right)}{5000 p_{3} \varrho_{2}(t)}
\end{array}\right] .
$$

The designed LTVLO for Bergman's model with the form (15) is:

$$
\begin{aligned}
& \dot{\hat{x}}_{1}=-\hat{x}_{1} \hat{x}_{2}+d(t)+l_{1}(t)\left(y-\hat{x}_{1}\right) \\
& \dot{\hat{x}}_{2}=-p_{2} \hat{x}_{2}+p_{3} \hat{x}_{3}-p_{3} I_{b}+l_{2}(t)\left(y-\hat{x}_{1}\right) \\
& \dot{\hat{x}}_{3}=-\eta \hat{x}_{3}+\eta I_{b}+u+l_{3}(t)\left(y-\hat{x}_{1}\right)
\end{aligned}
$$

, where the parameters $p_{2}, p_{3}, \eta$ and $I_{b}$ corresponds to the mean of the parameters of three patients shown in Table 1 ; with all the elements of formulation (15) the unmeasurable state variables $\hat{x}$ for T1DM Bergman's model (1) are estimated.

The same procedure explained for Bergman's model (1) is applied to Hovorka's model (4) (Stages I and II of subsection III-A). For simplicity, the description of this procedure applied to Hovorka's model is not shown. The asymptotic Luenberger observer is initialized around $\pm 10 \%$ of initial values of VP. This is due to biological conditions of VP which allows faster convergence.

\section{EXPERIMENTS DESIGN}

In this subsection, the experiments design explanation is done for each T1DM model described in this work. We proposed three experiments: A) VP modeled with Bergman's model (1) and LTVLO with Bergman's model structure, B) VP modeled with Hovorka's model (4) and LTVLO with Hovorka's model structure, and C) data come from real patients and observer is with Hovorka's model structure.

\section{1) Experiments definition}

The implementation, as models and as observers, is done in the continuous time domain, using numerical integration with fixed step-size $=1 s$, and with Runge Kutta solver. In order to obtain the results, Matlab $b^{T M}$ and Simulink ${ }^{T M}$ are used. The total time simulation is $48 \mathrm{hrs}$ with the beginning at 00:00 hrs of the first day; the time used for data analysis is $24 \mathrm{hrs}$, which starts at 00:00 hrs of the second day and ends at 23:59hrs the same day. The first day includes the transient period; for practical effects of the present LTVLO, this transient is not shown. The meal plan was identical for all VP. The plan was composed of: breakfast at 7:30 hrs, $40 \mathrm{~g}$ of CHOs; snack at 11:00hrs, $15 \mathrm{~g}$ of CHOs; lunch at 13:00 hrs, $90 \mathrm{~g}$ of CHOs; dinner at 19:00 hrs, $80 \mathrm{~g}$ of CHOs; and snack at 23:00hrs, $15 \mathrm{~g}$ of CHOs [38]. The prandial bolus was fitted to each patient with his insulin to CHO ratio (ICR) and administered fifteen minutes before of the meal intake. The ICR was calculated with the criterion to avoid the postprandial hypoglycaemia to each patient, the snacks do not have prandial insulin boluses. In order to demonstrate the observer convergence ability estimating the state variables, three different experiments are proposed, which are described as follows:

A) Bergman in silico model involves three VP. The LTVLO is designed with Bergman's model structure (1) and parametrized with a mean of three VP shown in Table 1 as VP\#0. First, the LTVLO methodology is applied to Bergman's model because it is simpler to understand. The observer is tested to estimate the variables of the three different patients.

B) Hovorka in silico model implies six VP. Then, the approach of a time-varying observer is extended to a more complex system, as it is Hovorka's model (4). The observer is parametrized with values of VP\#0 from Table 2.

C) Estimation of the inner variables from real patients. Data were obtained from a clinical study registered with ClinicalTrial.Gov, number NCT02160184 [39]. The clinical study treats about a glucose control algorithm in real T1DM patients, where it is known continuous glucose measurements, insulin doses and amount of ingested CHOs per meal, and with this information, the LTVLO estimates the corresponding Hovorka's variables. Each patient was equipped with a CGM device (Paradigm $^{\circledR}$ REAL-Time; Medtronic, Minneapolis, MN) and an insulin pump Animas ${ }^{\circledR} 2020$ (Animas Corp., West Chester, PA). The measurement period and infusion period was 5 minutes. The real patient data is stored in a data structure which feeds with the available information (glucose measurements, insulin doses, carbohydrate meal) the LTVLO. The 
LTVLO remains the same as designed in experiment B with Hovorka's model structure and parameterized with values of VP\#0 in Table 2.

\section{2) Experiments evaluation}

The results representation exhibit a qualitative performance of LTVLO. However, it is necessary to employ a quantitative parameter. In this sense, the parameter fit of estimated variables to model variables offer a quantitative reliability of estimation capacities of the designed Luenberger observer. The fit parameter expressed in percentage is calculated for every state as:

$$
\text { fit }=\sqrt{1-\frac{\sum_{t=1}^{N}[x(t)-\hat{x}(t)]^{2}}{\sum_{t=1}^{N}[x(t)-\bar{x}]^{2}}} \times 100 \%,
$$

where $N$ is the number of iterations that the simulation executes, $x(t)$ are the state space variables of the model and $\hat{x}(t)$ are the corresponding estimated variables [40].

\section{RESULTS}

In this section, it is shown the performance of the LTVLO. For simplicity, only $24 \mathrm{hrs}$ of simulation time are shown. The initial time of simulation corresponds to 00:00 hrs of the second day.

\section{A. EXPERIMENT A: ESTIMATION OF BERGMAN VARIABLES}

The proposed methodology is applied to design an observer to Bergman's model (1), whose parameters are set by the mean of the model parameters (patient zero). The LTVLO is tested with VP generated by parameters of the three patients described in [29].

Figure 2 shows the three states of VP\#1, estimated states, and their corresponding time-varying gains when the meal plan is applied. Each observer variable estimates asymptotically its corresponding model variable in approximately $100 \mathrm{~s}$ of simulation, tracking them for the rest of simulation. The states $X\left(x_{2}\right)$, insulin influence and $I\left(x_{3}\right)$, plasma insulin, are increased due to insulin dose applied at 7:15 hrs. When the VP\#1 is having breakfast at 7:30 hrs, the state $G$ $\left(x_{1}\right)$ plasma glucose, is increased due to CHOs intake. The gains $l_{1}(t), l_{2}(t)$ and $l_{3}(t)$ change suddenly when the model variables present high rate of change.

Table 3, shows the reliability percentage of estimated variables by the LTVLO; having over $94 \%$ of confidence on the three observed variables. These results represent an accurate estimation of the non-measurable variables.

TABLE 3. fit (\%) of LTVLO to Bergman's model.

\begin{tabular}{cccc}
\hline Variable renamed & $x_{1}$ & $x_{2}$ & $x_{3}$ \\
\hline VP\#1 & 100 & 99.99 & 94.31 \\
VP\#2 & 100 & 99.99 & 99.68 \\
VP\#3 & 100 & 99.99 & 97.78 \\
\hline
\end{tabular}

\section{B. EXPERIMENT B: ESTIMATION OF HOVORKA VARIABLES}

The proposed methodology is applied to develop an observer to Hovorka's model (4), which is parameterized with the zero-patient values (mean of six patient parameters included in [33]). The LTVLO is tested with the other six VP generated from the parameters in [33].

Figure 3 shows three state variables $\left(x_{1}, x_{3}\right.$ and $\left.x_{7}\right)$ of Hovorka's model, in particular related to VP\#6, the behaviour of the observer states estimated, and their corresponding time-varying gains, when the meal plan is applied. The states $x_{3}$, non-accessible glucose $\left(Q_{2}\right)$ and $x_{7}$, plasma insulin $(I)$, are increased due to insulin dose applied at 7:15 $\mathrm{hrs}$. When the VP\#6 is having breakfast at 7:30 hrs, the state $x_{1}$ subcutaneous glucose $(C)$, is increased due to CHOs intake. The gains $l_{1}(t), l_{3}(t)$ and $l_{7}(t)$ change suddenly when the model variables present high rate of change. Even when the states observer variables $\left(x_{3}\right.$ and $\left.x_{7}\right)$ do not match accurately the state model variables $\left(Q_{2}\right.$ and $\left.I\right)$, qualitatively have a similar profile as their corresponding model variable. This is due to the different parametrization of VP\#6 and the LTVLO. Table 4 shows the reliability percentage of estimated variables by the Luenberger time-varying observer to Hovorka's model.

The $f i t$ of the estimation variables $x_{1}, x_{3}$ and $x_{7}$ for the six patients is between the values of $31.27 \%$ and $100 \%$. This is explained because these variables are directly influenced by known information, glucose, insulin, and CHOs absorption via gut. Variable $x_{2}$ for VP\#3 is $79.45 \%$, which can be due to the parametrization of the LTVLO. As it can be seen in Figure $3 \mathrm{c}$ ), the estimation is not as accurate as it seems to be. Nevertheless, its corresponding fit value for patient six, is acceptable $(88.88 \%)$. In general, fit values between $50 \%$ and $90 \%$ can be associated with the extremely sensitive metric of the fit parameter to differentiate between means of estimated variables and model variables [40]. The fit value for variable $x_{3}$ for patient $4(31.27 \%)$ corresponds to a poor estimation of this variable.

\section{EXPERIMENT C: ESTIMATING REAL T1DM PATIENTS UNMEASURABLE VARIABLES}

The LTVLO is employed to estimate the corresponding Hovorka model state variables from real patient data, for simplicity, are shown results only for a set of patient data. Figure 4 a) displays the estimation of variable $x_{1}$ by its corresponding time-varying observer variable, the glucose measured with the sensor having a fit $=99.61 \%$; b) shows the corresponding gain evolution. c) and e) correspond to unmeasurable variables of non-accessible glucose compartment and plasma insulin, respectively. Frame d) is the gain adjustment for the non-accessible glucose compartment and f) is the shape depicting the evolution at each iteration of Luenberger gain for the plasma insulin. The CHOs intake of this particular real patient is depicted with black triangles, corresponding to $20 \mathrm{~g}$ at $7: 00 \mathrm{hrs}, 60 \mathrm{~g}$ at $10: 00 \mathrm{hrs}, 80 \mathrm{~g}$ at 15:15 hrs and $80 \mathrm{~g}$ at $21 \mathrm{hrs}$. 

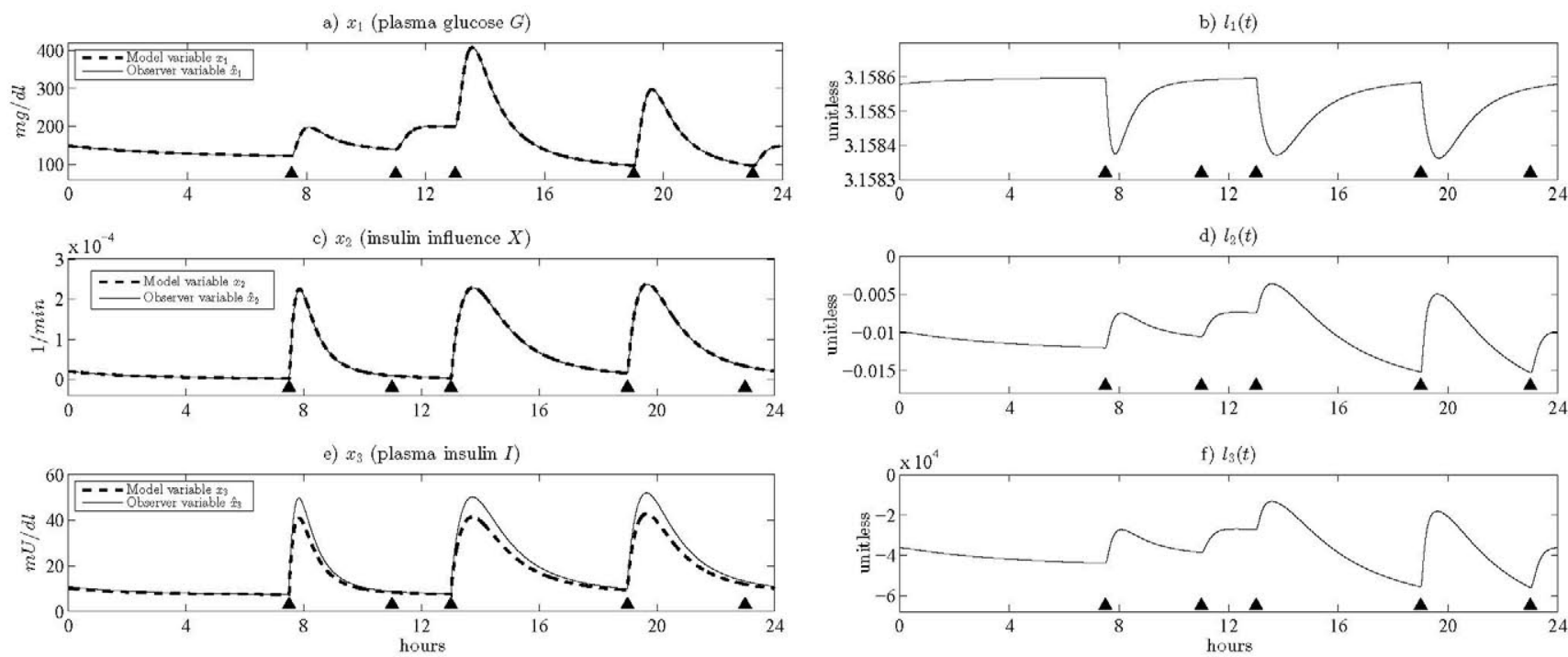

FIGURE 2. VP\#1 states from Bergman's model, states estimated from LTVLO and their respective time-varying gains: a) comparison of plasma glucose $x_{1}$ vs. $\hat{x}_{1}$; b) gain $l_{1}(t)$ relative to $\hat{x}_{1} ;$ c) comparison of insulin influence $x_{2}$ vs. $\hat{x}_{2} ;$ d) gain $l_{2}(t)$ relative to $\hat{x}_{2} ;$ e) comparison of plasma insulin $x_{3}$ vs. $\hat{x}_{3} ;$ f) gain $l_{3}(t)$ relative to $\hat{x}_{3}$. The triangles show the moment in which the meal is ingested by the patient according to the meal plan.

a) $x_{1}$ (subcutaneous glucose $C$ )

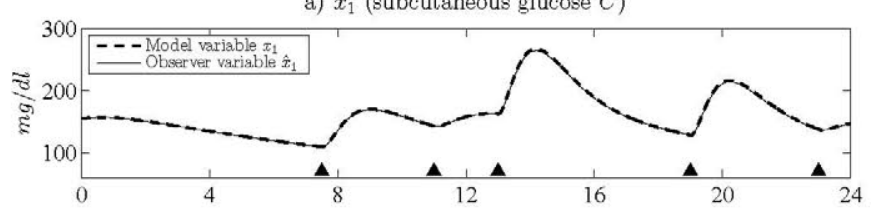

c) $x_{3}$ (non-accesible glucose $Q_{2}$ )

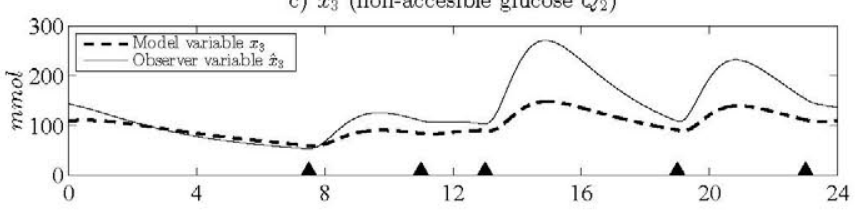

e) $x_{7}$ (plasma insulin $I$ )

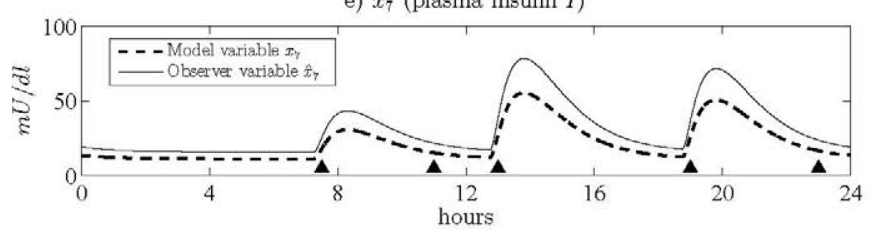

b) $l_{1}(t)$

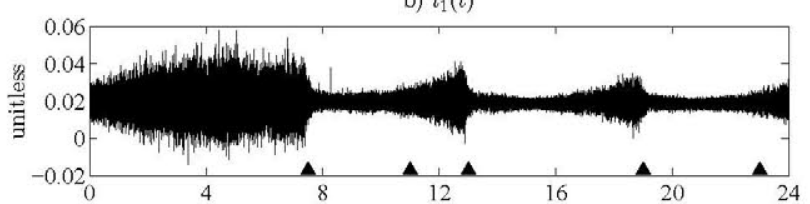

d) $l_{3}(t)$

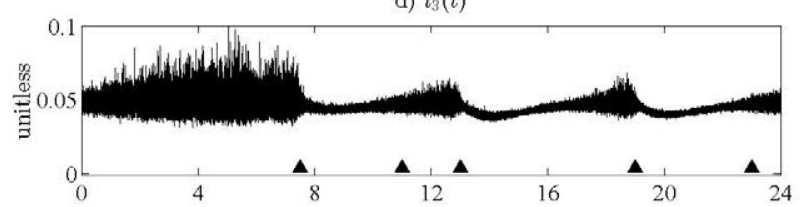

f) $l_{7}(t)$

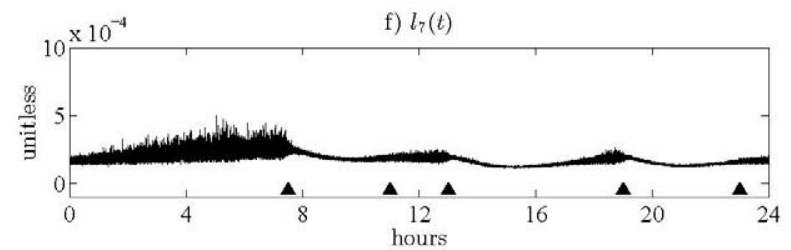

FIGURE 3. VP\#6 states from Hovorka's model, states estimated from Luenberger time-varying observer and their respective time-varying gains: a) comparison of CGM sensor $x_{1}$ vs. $\hat{x}_{1} ;$ b) gain $l_{1}(t)$ relative to $\hat{x}_{1} ;$ c) comparison of non-accessible glucose $x_{3}$ vs. $\hat{x}_{3} ;$ d) gain $l_{3}(t)$ relative to $\hat{x}_{3} ;$ e) comparison of plasma insulin $x_{7}$ vs. $\left.\hat{x}_{7} ; \mathrm{f}\right)$ gain $l_{7}(t)$ relative to $\hat{x}_{7}$.

TABLE 4. fit (\%) of LTVLO to Hovorka's model.

\begin{tabular}{cccccccccc}
\hline Variable renamed & $x_{1}$ & $x_{2}$ & $x_{3}$ & $x_{4}$ & $x_{5}$ & $x_{6}$ & $x_{7}$ & $x_{8}$ & $x_{9}$ \\
\hline VP\#1 & 99.35 & 92.77 & 58.68 & 98.59 & 99.88 & 68.45 & 73.23 & 99.98 & 100 \\
VP\#2 & 99.99 & 92.97 & 97.03 & 94.86 & 82.49 & 70.97 & 91.04 & 99.98 & 100 \\
VP\#3 & 99.90 & 79.45 & 95.43 & 96.98 & 94.18 & 94.07 & 88.15 & 99.99 & 100 \\
VP\#4 & 99.99 & 99.46 & 31.27 & 94.66 & 98.19 & 61.45 & 60.74 & 99.99 & 100 \\
VP\#5 & 98.94 & 99.65 & 90.20 & 99.74 & 64.51 & 69.80 & 55.89 & 99.98 & 100 \\
VP\#6 & 99.96 & 96.55 & 88.88 & 99.14 & 95.52 & 99.22 & 55.07 & 99.98 & 100 \\
\hline
\end{tabular}



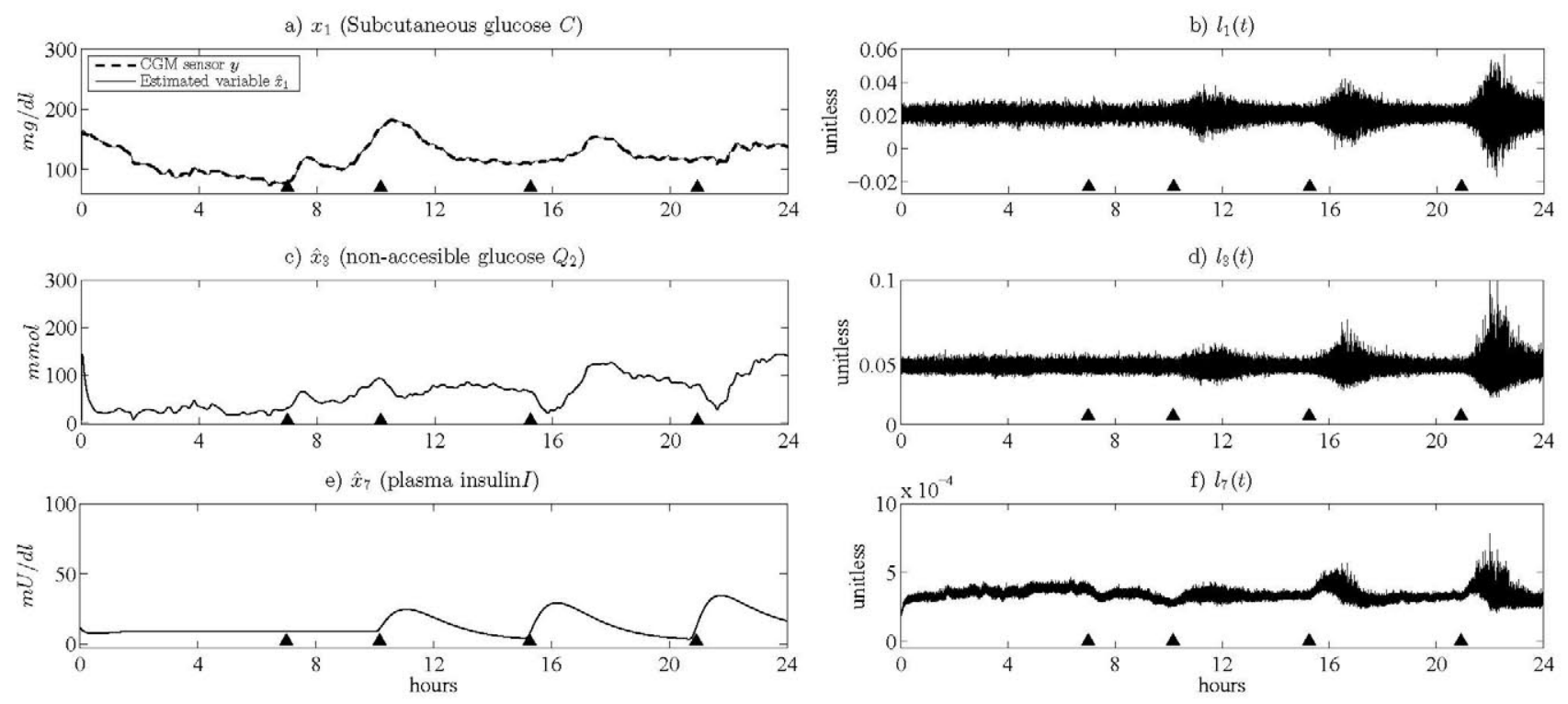

FIGURE 4. Estimation of the inner variables from real patients, states estimated from Luenberger time-varying observer and their respective time-varying gains: a) comparative of subcutaneous glucose $x_{1}$ vs. $\hat{x}_{1} ;$ b) gain $l_{1}(t)$ relative to $\left.x_{1} ; \mathrm{c}\right)$ non-accessible glucose estimation $\left.\hat{x}_{3} ; \mathrm{d}\right)$ gain $l_{3}(t)$ relative to $\left.x_{3} ; \mathrm{e}\right)$ plasma insulin estimation $\left.\hat{x}_{7} ; \mathrm{f}\right)$ gain $l_{7}(t)$ relative to $x_{7}$.

\section{DISCUSSION}

The LTVLO provides helpful information about unavailable variables to be measured in real-time so that they can be used as the base of a control law design in a future work. We have used the Luenberger methodology to build a T1DM observer; although, there are other methods as sliding modes observers or artificial neural networks. Luenberger methodology is easy to understand and allows selecting the desired dynamic of the estimated variables.

Even when linear time-varying systems result in a challenge to guarantee the observability condition, we guarantee such condition in each time instant. Although, for the particular cases considered in this work, the observability condition depends on the parameter values, which define the VP dynamics and are determined by the author of the model. These parameter values characterized the model dynamics and are used to design the observer, but we could explore other parameters; e.g. setting the VP parameters on the observer according to real patient behaviour classifying them by their insulin sensitivity.

The location of desired observer eigenvalues $\lambda_{n}$ to the left of eigenvalues of the matrix $A(t)$ at $t_{0}$, results in a good practice assuring faster convergence of the observer and to consider that the system response is dominated by the observer eigenvalues. The best location of the eigenvalues is out of the scope of this paper.

The operation point $\rho$ is chosen from a basal period ( $y=$ $90 \mathrm{mg} / \mathrm{dl}$ ), with the aim of having the desired dynamic on the patient when this one is not disturbed and the linearization of the model is made concerning a stable operating point.

We have chosen the fit parameter to evaluate the performance of the observer. This is because it is a percentage measurement defined independently of the variable scale versus the mean square error.

In experiment A) the fit parameter indicates a high precision estimation mainly due to the simplicity of Bergman's model. There is only one variable between the interaction of the output variable (blood glucose) and the variable that contains the input (insulin infusion), which facilitates the calculus of the Luenberger gains. The plasma glucose $G\left(x_{1}\right)$ is estimated with $100 \%$, and the plasma insulin $I\left(x_{3}\right)$ above $94 \%$.

In experiment B) the variables $C\left(x_{1}\right), Q_{2}\left(x_{3}\right)$ and $I\left(x_{7}\right)$ were chosen in order to reduce the space used demonstrating how the LTVLO reduce the estimation error for the measurable variable, and it is able to estimate the unmeasurable variables with the established parametrization. Luenberger gains show variation due to the high rate of updating made at each simulation instant; this is explained because once the observer reduces at a minimum the estimation error of the measurable variable (subcutaneous glucose), the Luenberger gains are varying upon a mean.

As it can be seen on Table 4, the fit parameter for patient 4 of variable $Q_{2}\left(x_{3}\right)$, the value $31.27 \%$, quantitatively is indicating that the observer has low reliability because it is not estimating well its corresponding variable. Nevertheless, qualitatively is seen that estimated variables by the observer, are conditioned to the parametrization of LTVLO. This parametrization causes the existence of differences between VP variables profiles and the estimated variables.

The reliability in estimated variables by LTVLO to Hovorka VP in $C\left(x_{1}\right)$ is above $98 \%$ but in state $I\left(x_{7}\right)$ is over $55 \%$ because the observer always proposed a high value of $\hat{x}_{7}$. This result can be good when it is intended to design 
a control law because the insulin excess can condition the control behaviour to do it less sensible and therefore avoid the hypoglycaemic events. From information provided in Table 4 , it can be seen that state variable $x_{2}$ would be used as the main variable to feedback a control law because the plasma glucose is the desired variable to control.

Table 5 summarizes the minimum values of the fit parameter shown in Tables 3 and 4. The minimum values of the variables are grouped by their physiological relation.

TABLE 5. Summary of minimum fit (\%) to VP population.

\begin{tabular}{|c|c|c|c|c|c|c|}
\hline & \multicolumn{3}{|c|}{$\begin{array}{c}\text { Bergman LTVLO performance } \\
\text { (From Table 3) }\end{array}$} & \multicolumn{3}{|c|}{$\begin{array}{l}\text { Hovorka LTVLO performance } \\
\text { (From Table 4) }\end{array}$} \\
\hline & Variable & fit $(\%)$ & Patient & Patient & fit $(\%)$ & Variable \\
\hline \multirow{3}{*}{ 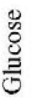 } & & & & 5 & 98.94 & $x_{1}$ \\
\hline & $x_{1}$ & 100 & All & 3 & 79.45 & $x_{2}$ \\
\hline & & & & 4 & 31.27 & $x_{3}$ \\
\hline \multirow{3}{*}{ 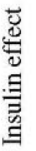 } & & & & 4 & 94.66 & $x_{1}$ \\
\hline & $x_{2}$ & 99.99 & All & 5 & 64.51 & $x_{5}$ \\
\hline & & & & 4 & 61.45 & $x_{6}$ \\
\hline \multirow{3}{*}{ 㫪 } & & & & 6 & 55.07 & $x_{7}$ \\
\hline & $x_{3}$ & 94.31 & 1 & $1,2,5,6$ & 99.98 & $x_{8}$ \\
\hline & & & All & & 100 & $x_{9}$ \\
\hline
\end{tabular}

As it can be seen in Table 5, the values of glucose compartments for Bergman and Hovorka present high accuracy. The intermediate insulin effects compartments and plasma insulin compartment are the inaccuracy fit results of the LTVLO, because the observer always adjusted a high value to these variables. To Hovorka LTVLO results, the insulin compartments and glucose compartments have the better fit performance because these state variables are directly connected with available information.

The fit values between $90 \%$ and $100 \%$ constitute a very accurate estimation, which means that the estimation error $e \approx 0$. A fit value between $50 \%$ and $90 \%$ means that the correlation error between the model variable and the estimated variable is increased in some periods. The fit value between $1 \%$ and $50 \%$ means that the coincidence between the model variable and estimated variable occurs during small periods and the majority of the time there are differences between them. A fit value of $0 \%$ means that the estimated variable does not match its corresponding variable, but the LTVLO is providing helpful information biased by its parametrization.

In experiment $\mathrm{C}$ ) the unmeasurable variables are estimated under a well-known model structure, although the measurement by the CGM is noisy. The model structure matches the output variable (subcutaneous glucose) and input variable (insulin dose) with the data available from real patients. With Hovorka's model structure on the LTVLO, it is possible to incorporate information about the main disturbances (CHOs intake) available with the patients data. The chosen variables $C, Q_{2}$ and $I$ graphed for this experiment demonstrate how the LTVLO reduce the estimation error for the measurable variable and it is able to estimate the unmeasurable variables.
However, we do not know the CGM sensor accuracy. The measured variable by a CGM is objective because comes from a sensor. Both meal and doses are recorded by the patients and sometimes they forget to write down some data. The LTVLO in front of a CGM sensor calibration or loss of information becomes oscillating or unstable. For this reason, the LTVLO needs to be strengthened against those adversities to be applied in a real scenario with T1DM patients.

\section{CONCLUSION}

This paper proposes a Luenberger time-varying observer applied to Bergman and Hovorka T1DM models. The observer has the feature of being updated each time instant in order to estimate the states under a known structure, and only with the knowledge of the insulin dose, the CGM information and the intake of CHOs. Lyapunov stability proof establishes the operation boundedness of the time-varying proposal. The results obtained in the designed experiments validate the applicability of the proposed observer. Additionally, a timevarying observer used to estimate the unmeasurable variables with a well-known structure, opens a possibility to apply a control law (employed in other areas with success) in order to improve the design and development of the ambulatory artificial pancreas.

\section{APPENDIX A PROOF OF THEOREM 1}

Proof: Let $e=0$ be an equilibrium point asymptotically stable for the Luenberger time-varying system (15) represented in the error dynamic (26). Lyapunov's candidate function positive definite is described in terms of the error as:

$$
V(e, t)=e^{\top} P(t) e,
$$

where $\mathbf{P}(t)$ is continuously differentiable, bounded and positive definite symmetric matrix [41]. The derivative of Lyapunov candidate function is computed as:

$$
\dot{V}(e, t)=e^{\top} P(t) \dot{e}+\dot{e}^{\top} P(t) e+e^{\top} \dot{P}(t) e,
$$

where $\dot{P}(t)$ must be negative definite to guarantee that function (36) is negative definite. Using the Riccati's function as:

$$
\dot{P}(t)=-P(t) \boldsymbol{\Lambda}(t)-\boldsymbol{\Lambda}(t)^{\top} P(t)-Q(t),
$$

for $\dot{P}(t)=0$ and $\boldsymbol{\Lambda}(t)=[A(t)-l(t) c]$ from $(26), \mathbf{Q}(\mathrm{t})$ must be continuous, positive definite and symmetric. Then, it is computed the matrix $P(t)$ such that fulfill the aforementioned characteristics. Now replacing (26) and (37) into (36), yields:

$$
\dot{V}(e, t)=-e^{\top} Q(t) e+\left(d-d_{1}\right),
$$

which is negative definite with $\left|e^{\top} Q(t) e\right| \geq\left|d-d_{1}\right|$, showing that the LTVLO (15) in the error dynamics (26) is an asymptotically stable estimator. The derivative of $V(e, t)$ along the trajectories of (26) is given by:

$$
\dot{V}(e, t)=\frac{\partial V}{\partial t}+\frac{\partial V}{\partial e} f(e, t)+\frac{\partial V}{\partial e} g(e, t),
$$


where $f(e, t)$ are the state space functions in the error dynamic and $g(e, t)$ are the functions related to the disturbances. Then, applying the derivative along the trajectories (39), it is obtained:

$$
\dot{V}(e, t)=-\|e\|_{1}^{2}+2 l(t) e_{1}^{2} \varphi_{f}+2\left(d-d_{1}\right) e_{1} \varphi_{g},
$$

where $\varphi_{f}$ and $\varphi_{g}$ correspond to the coefficients of second and third terms of equation (39), respectively. Suppose $l(t) \leq \frac{\zeta-1}{-2 K^{2}}$, where $0 \leq \zeta \leq 1 \forall\left|e_{1}\right| \leq K$, and using the inequality $\left(d-d_{1}\right) \leq \delta$, where $\delta$ is the maximum value of $\left(d-d_{1}\right)$ and $\varphi_{g} e_{1} \leq\|e\|_{1}\left|\varphi_{g}\right|$, yields:

$$
\dot{V}(e, t) \leq-\zeta\|e\|_{1}^{2}+2 \delta\left|\varphi_{g}\right|\|e\|_{1}^{2},
$$

where $\zeta \leq\left(1-2 l(t) K^{2}\right) \forall\left|e_{1}\right| \leq K$, with $K$ as the upper bound on $\left|e_{1}\right|$. Suppose that:

$$
\theta \leq \frac{2 \delta\left|\varphi_{g}\right|}{\zeta|| e \|_{1}} .
$$

Then

$$
\dot{V}(e, t) \leq-(1-\theta) \zeta\|e\|_{1}^{2},
$$

where $0 \leq \theta \leq 1$. From (42), it is established that:

$$
\|e\|_{1} \leq \mu=\frac{2 \delta\left|\varphi_{g}\right|}{\zeta \theta} .
$$

In order to estimate the bound $K$, let $\Omega_{c}=\{e \in$ $\left.\Re^{n} \mid V(e, t) \leq c\right\}$. For any positive constant $c$, the set $\Omega_{c}$ is closed and bounded. The boundary of $\Omega_{c}$ is the Lyapunov surface $V(e, t)$. The largest value of $\left|e_{1}\right|$ on the surface $V(e, t)=c$, can be determined by differentiating the surface equation partially with respect to $e_{q}$, where $q=2, \ldots, n$ (for Bergman's Model $n=3$ and for Hovorka's model $n=9$ ), as:

$$
\frac{\partial V}{\partial e_{q}}=0 .
$$

By simple calculations to evaluate the Lyapunov candidate function with the partial derivatives, it is concluded that:

$$
\left.V(e, t)\right|_{\frac{\partial V}{\partial e_{q}=0}}=\Upsilon\left(e_{1}, t\right) .
$$

Then, the largest value of $e_{1}^{2}$ on the Lyapunov surface is $\frac{c}{r}$. Therefore, all points inside $\Omega_{c}$ satisfy the bound $\left|e_{1}\right| \leq K$, where $K^{2}=\frac{c}{\Upsilon}$.

Thus, if $l(t) \leq \frac{\Upsilon(\zeta-1)}{2 c}$ and $\delta$ is so small that $\mu^{2} \lambda_{\max }(P(t))<c$, then $B_{\mu} \subset \Omega_{c}$ and all trajectories starting inside $\Omega_{c}$ remain for all future time in $\Omega_{c}$. Therefore, the solutions of the disturbed system are uniformly ultimately bounded by:

$$
B=\mu \sqrt{\frac{\lambda_{\max }(P(t))}{\lambda_{\min }(P(t))}} .
$$

It is important to mention that $l(t)$ and $\left(d-d_{1}\right)$ are treated differently as two disturbance terms, since the first term vanishes at the origin, while the second one does not.

\section{REFERENCES}

[1] N. Pørksen, The in vivo regulation of pulsatile insulin secretion, Diabetologia 45 (1) (2002) 3-20.

[2] G. Grunberger, The need for better insulin therapy, Diabetes, Obesity and Metabolism 15 (s1) (2013) 1-5.

[3] D. Control, C. T. R. Group, et al., The effect of intensive treatment of diabetes on the development and progression of long-term complications in insulin-dependent diabetes mellitus, N Engl j Med 1993 (329) (1993) 977-986.

[4] N. Jeandidier, J.-P. Riveline, N. Tubiana-Rufi, A. Vambergue, B. Catargi, V. Melki, G. Charpentier, B. Guerci, Treatment of diabetes mellitus using an external insulin pump in clinical practice, Diabetes \& metabolism 34 (4) (2008) 425 438.

[5] R. Hovorka, M. E Wilinska, L. J Chassin, C. L Acerini, D. B Dunger, The artificial pancreas: making headway, Practical Diabetes International 24 (2) (2007) 56-58.

[6] D. Lunn, C. Wei, R. Hovorka, Fitting dynamic models with forcing functions: application to continuous glucose monitoring in insulin therapy, Statistics in medicine 30 (18) (2011) 2234-2250.

[7] S. Trevitt, S. Simpson, A. Wood, Artificial pancreas device systems for the closed-loop control of type 1 diabetes: what systems are in development?, Journal of diabetes science and technology 10 (3) (2016) 714-723.

[8] R. N. Bergman, Y. Z. Ider, C. R. Bowden, C. Cobelli, Quantitative estimation of insulin sensitivity., American Journal of Physiology-Endocrinology And Metabolism 236 (6) (1979) E667-E677.

[9] J. T. Sorensen, A physiologic model of glucose metabolism in man and its use to design and assess improved insulin therapies for diabetes, Ph.D. thesis, Massachusetts Institute of Technology (1985).

[10] R. Hovorka, V. Canonico, L. J. Chassin, U. Haueter, M. Massi-Benedetti, M. O. Federici, T. R. Pieber, H. C. Schaller, L. Schaupp, T. Vering, et al., Nonlinear model predictive control of glucose concentration in subjects with type 1 diabetes, Physiological measurement 25 (4) (2004) 905-920.

[11] C. Dalla Man, R. A. Rizza, C. Cobelli, Meal simulation model of the glucose-insulin system, IEEE Transactions on Biomedical Engineering 54 (10) (2007) 1740-1749.

[12] P. Palumbo, P. Pepe, S. Panunzi, A. De Gaetano, Time-delay modelbased control of the glucose-insulin system, by means of a state observer, European Journal of Control 18 (6) (2012) 591-606.

[13] P. Home, Plasma insulin profiles after subcutaneous injection: how close can we get to physiology in people with diabetes?, Diabetes, Obesity and Metabolism 17 (11) (2015) 1011-1020.

[14] F. Fichera, C. Prieur, S. Tarbouriech, L. Zaccarian, Using luenberger observers and dwell-time logic for feedback hybrid loops in continuous-time control systems, International Journal of Robust and Nonlinear Control 23 (10) (2013) 1065-1086.

[15] V. N. Phat, N. T. Thanh, H. Trinh, Full-order observer design for nonlinear complex large-scale systems with unknown time-varying delayed interactions, Complexity 21 (2) (2015) 123-133.

[16] M. Heydari, M. A. Demetriou, Distributed kalman filters with adaptive strategy for linear time-varying interconnected systems, International Journal of Adaptive Control and Signal Processing (2015) 1-15.

[17] A.-G. Wu, G. Feng, G.-R. Duan, Proportional multiple-integral observer design for discrete-time descriptor linear systems, International Journal of Systems Science 43 (8) (2012) 1492-1503.

[18] M.-S. Chen, S.-Y. Lin, M.-L. Tseng, Y.-L. Yeh, J.-Y. Yen, Robust stateand-disturbance observer design for linear non-minimum-phase systems, Asian Journal of Control (2015) 1135-1141.

[19] F. Mazenc, O. Bernard, Interval observers for linear time-invariant systems with disturbances, Automatica 47 (1) (2011) 140-147.

[20] W.-J. Liu, Adaptive sliding mode observer design for a class of uncertain systems, International Journal of Adaptive Control and Signal Processing 28 (12) (2014) 1341-1356.

[21] D. Mincarelli, A. Pisano, T. Floquet, E. Usai, Uniformly convergent sliding mode-based observation for switched linear systems, International Journal of Robust and Nonlinear Control (2015) 1549-1564.

[22] J. Zhang, A. K. Swain, S. K. Nguang, Robust sliding mode observer based fault estimation for certain class of uncertain nonlinear systems, Asian Journal of Control 17 (4) (2015) 1296-1309.

[23] W. Chen, F. N. Chowdhury, A synthesized design of sliding-mode and luenberger observers for early detection of incipient faults, International Journal of Adaptive Control and Signal Processing 24 (12) (2010) 10211035 . 
[24] G. Xia, X. Shao, A. Zhao, Robust nonlinear observer and observerbackstepping control design for surface ships, Asian Journal of Control 17 (4) (2015) 1377-1393.

[25] P. Palumbo, G. Pizzichelli, S. Panunzi, P. Pepe, A. De Gaetano, Modelbased control of plasma glycemia: Tests on populations of virtual patients, Mathematical biosciences Vol. 257 (2014) 2-10.

[26] L. Kovács, B. Paláncz, Z. Benyó, Design of luenberger observer for glucose-insulin control via mathematica, in: 2007 29th Annual International Conference of the IEEE Engineering in Medicine and Biology Society, IEEE, 2007, pp. 624-627.

[27] P. Palumbo, P. Pepe, S. Panunzi, A. De Gaetano, Observer-based closedloop control for the glucose-insulin system: local input-to-state stability with respect to unknown meal disturbances, in: 2013 American Control Conference, IEEE, 2013, pp. 1751-1756.

[28] A. Borri, F. Cacace, A. De Gaetano, A. Germani, C. Manes, P. Palumbo, S. Panunzi, P. Pepe, Luenberger-like observers for nonlinear time-delay systems with application to the artificial pancreas: The attainment of good performance, IEEE Control Systems 37 (4) (2017) 33-49.

[29] P. Kaveh, Y. B. Shtessel, Blood glucose regulation via double loop higher order sliding mode control and multiple sampling rate, in: Modern Sliding Mode Control Theory, Springer, 2008, pp. 427-445.

[30] M. Berger, D. Rodbard, Computer simulation of plasma insulin and glucose dynamics after subcutaneous insulin injection, Diabetes care 12 (10) (1989) 725-736.

[31] E. Lehmann, T. Deutsch, A physiological model of glucose-insulin interaction in type 1 diabetes mellitus, Journal of biomedical engineering 14 (3) (1992) 235-242.

[32] A. Rodríguez-Herrero, C. Pérez-Gandía, M. Rigla, A. de Leiva, E. J. Gómez, M. E. Hernando, A simulation study of an inverse controller for closed-and semiclosed-loop control in type 1 diabetes, Diabetes technology \& therapeutics 12 (2) (2010) 95-104.

[33] R. Hovorka, F. Shojaee-Moradie, P. V. Carroll, L. J. Chassin, I. J. Gowrie, N. C. Jackson, R. S. Tudor, A. M. Umpleby, R. H. Jones, Partitioning glucose distribution/transport, disposal, and endogenous production during ivgtt, American Journal of Physiology-Endocrinology and Metabolism 282 (5) (2002) E992-E1007.

[34] M. E. Wilinska, L. J. Chassin, C. L. Acerini, J. M. Allen, D. B. Dunger, R. Hovorka, Simulation environment to evaluate closed-loop insulin delivery systems in type 1 diabetes, Journal of diabetes science and technology 4 (1) (2010) 132-144.

[35] A. Isidori, Nonlinear control systems, Springer Science \& Business Media, 2000 .

[36] C.-T. Chen, Linear system theory and design, Oxford University Press, Inc., 1995.

[37] J. Ackermann, Der entwurf linearer regelungssysteme im zustandsraum, at-Automatisierungstechnik 20 (1-12) (1972) 297-300.

[38] C. Anguita, A. Caixás, J. Clapés, M. Claveria, I. Gallach, M. Jansà et al., Documento de consenso sobre recomendaciones nutricionales y de educación alimentaria en la diabetes: ACD 2003, Associació Catalana de Diabetis, 2003

[39] I. Capel, M. Rigla, G. García-Sáez, A. Rodríguez-Herrero, B. Pons, D. Subías, F. García-García, M. Gallach, M. Aguilar, C. Pérez-Gandía, et al., Artificial pancreas using a personalized rule-based controller achieves overnight normoglycemia in patients with type 1 diabetes, Diabetes technology \& therapeutics 16 (3) (2014) 172-179.

[40] D. A. Finan, H. Zisser, L. Jovanovic, W. C. Bevier, D. E. Seborg, Identification of linear dynamic models for type 1 diabetes: a simulation study, IFAC Proceedings Volumes 39 (2) (2006) 503-508.

[41] H. K. Khalil, Nonlinear systems, Vol. 3, Prentice Hall, Upper Saddle River, NJ 07458,2002 